Ann. Zootech., I980, 29 (3), 229-244.

I.N.R.A.

BIBLIOTHEQUE UO 359:
DOMAINE DE CROUELLE

63039

Clermant-FD CEDEX?

\title{
Alimentation et croissance des civelles d'Anguilla anguilla L. (Poisson Téléosteen-anguilliforme) élevées expérimentalement en "canalisation ", au laboratoire
}

\author{
P. ELIE et J. DAGUZAN \\ avec la collaboration technique de M. M. LE PIVER, \\ Laboratoire de Zoologie générale et d'Écophysiologie \\ Faculté des Sciences Biologiques, Université de Rennes I \\ Avenue du Général-Leclerc, 35042 Rennes Cedex (France)
}

\section{I. - Résumé}

La croissance des Civelles d'Anguilla anguilla $\mathrm{L}_{1}$. est étudiée dans un nouveau système d'élevage dit : " en canalisation ". Des charges biotiques de $10 \mathrm{~kg} / \mathrm{m}^{3}$ sont atteintes après $\mathrm{I} 22$ jours d'alimentation. La prise de nourriture, les comportements durant la phase d'alimentation et enfin l'activité et la localisation préféreritielle des Civelles sont analysés. Les différentes causes de mortalité sont pour la plupart mises en évidence.

\section{II. - Introduction}

De I960 à I968, la production des élevages d'Anguilla japonica (TEMunck et SCHLEGEL), au Japon, passe de 4800 tonnes à près de 24 ooo tonnes (dernier tonnage produit par I 682 exploitations) (MATSUI, I952; KOOPS, I966; QUERELLOU, I973; USUI, I974; FORREST, I976; TESCH, I977). A l'heure actuelle, cette production semble se stabiliser à 27000 tonnes environ.

De plus, en I 969 , le Japon commence à importer, en vue de l'élevage, des Civelles d'Anguilla anguilla L., mais entre 1970 et 1974 , les résultats obtenus sont très médiocres (taux de mortalité de 80 à 90 p. roo). Aujourd'hui, il semble que les paramètres de 1'élevage de la Civelle européenne soient mieux connus (ITOSHI, I972; CUEFF, I974; SEltz, I974; Kuhl,MANN, I975 et I976; ELIE, I975 et I979; ELIE et DAGUZAN, I976).

Les débuts de l'élevage de 1'Anguille en France datent de Ig68, sur une 
initiative de la compagnie des Salins du Midi et des Salines de 1'Est. Le C.T.G.R.F.F. (Centre Technique du Génie Rural des Eaux et Forêts) entreprend en mars r974, des études sur l'élevage des Civelles en circuit fermé (eau douce), et effectue des tests de croissance selon divers aliments existant sur le marché (SELTZ, I974).

En France, la médiocrité des résultats obtenus lors des différents essais est essentiellement due à trois facteurs limitants : socio-économiques, techniques et biologiques. Les échecs enregistrés par les salins du Midi et la Compagnie Générale Transatlantique semblent être les conséquences de l'utilisation de populations d'Anguillettes très hétérogènes en qualité et potentialité de croissance (QUERELLOU, I975). L'élevage de l'Anguille ne pourra donc se réaliser dans de meilleures conditions, que lorsque la maîtrise du grossissement des Civelles sera acquise. En effet, malgré les travaux sur la reproduction des Anguilles au laboratoire (FonTAINE, I936, I964; TUzet et Fontarne, I937; Boftrus, I962), le maintien des stades larvaires et la métamorphose ne sont pas encore possibles.

En ce qui nous concerne, nous avons montré récemment l'importance de la température sur l'alimentation et la croissance de la Civelle de l'Anguille européenne (Anguilla anguilla L.) et déterminé la température optimale de son élevage en eau saumâtre (EIIE et DAGUZAN, I976).

Devant le petit nombre de recherches effectuées sur l'élevage de la Civelle et à la suite de nos travaux, il semble intéressant de montrer l'importance du type de système d'élevage employé sur la croissance de la Civelle.

En effet, les structures d'élevage utilisées au Japon paraissent inadaptées au contexte socio-économique français : surface élevée, main d'œuvre importante, captures des individus difficiles, nécessitant deux journées avec Io personnes pour récupérer entre 80 et $95 \mathrm{p}$. Ioo de l'effectif présent dans un bassin d'élevage de $5000 \mathrm{~m}^{2}$.

Nous avons donc essayé de mettre au point une structure d'élevage (Unité d'élevage) mieux adaptée au contexte français et pouvant être facilement mise en œuvre (I).

\section{III. - Techniques expérimentales et matériel utilisé}

Le système utilisé permet d'effectuer un " élevage en canalisation ".

\section{A. - Caractéristiques de la "Canalisation" et de son circuit hydraulique}

Ce système d'élevage présente une capacité totale de 38 litres environ et comprend (fig. I et photo I) :

- un compartiment de chauffage et d'oxygénation de l'eau (A), réalisés à l'aide d'une électrode chauffante de $5_{5}$ o watts et d'une rampe d'arrivée d'eau sous pression;

- un compartiment d'élevage et d'oxygénation facultative (B) muni de deux micro-bulleurs permettant des réajustements de l'oxygénation de l'eau;

(I) Il faut noter que deux expérimentations analogues et simultanées ont été menées sur cette structure d'élevage. Devant les résultats similaires obtenus nous n'exposerons que les données relatives à l'une des expéricnces. 
- une canalisation obscure (C) servant d'abri pour les animaux;

- un compartiment d'alimentation (D);

- un compartiment d'évacuation de l'eau (E), assurée par un déversoir.
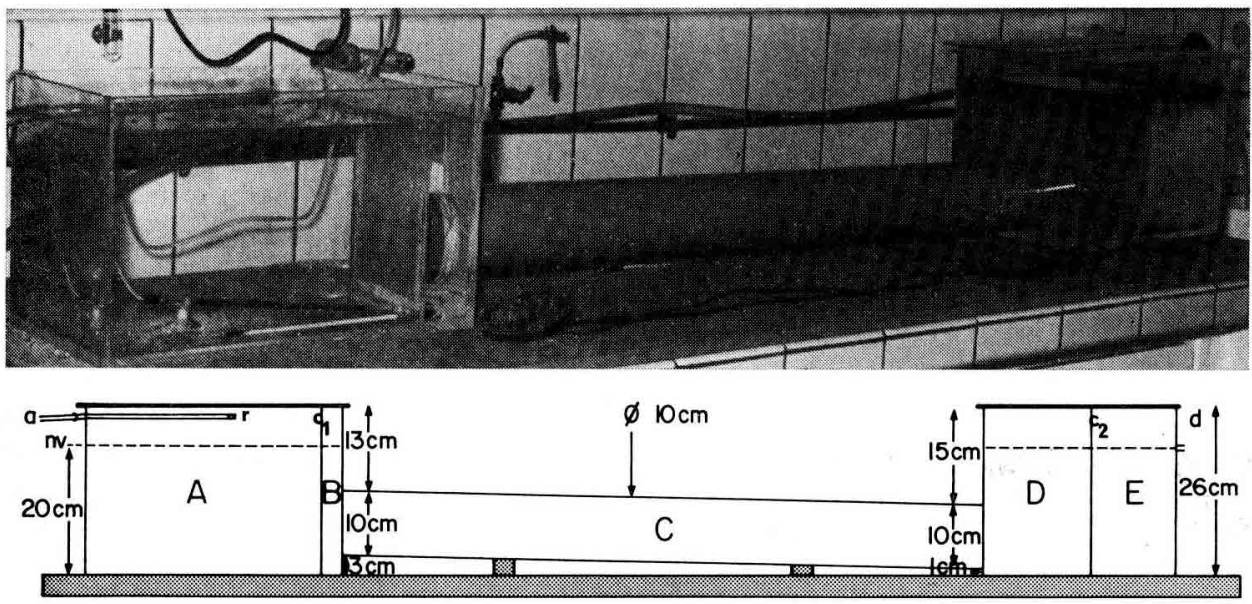

$36,5 \mathrm{~cm}$

$100 \mathrm{~cm}$

$16,5 \mathrm{~cm} \rightarrow-13,5 \mathrm{~cm}$

Fig. I. - Schéma et vue générale du système d'élevage en canalisation.

A : compartiment de chauffage et d'oxygénation

B : compartiment d'élevage et d'oxygénation

C : canalisation obscure

D : compartiment d'élevage et d'alimentation

E : compartiment d'évacuation de l'eau

$a$ : arrivée de l'eau

$\mathrm{C}_{1}$ et $\mathrm{C}_{2}$ : cloisons amovibles

$d$ : déversoir du trop plein d'eau

$n v$ : niveau de l'eau

$r$ : rampe d'oxygénation.

Scheme and general view of the rearing system in canalization.

A : heating and oxygenation compartment

$B$ : rearing and oxygenation compartment

C : dark canalization

$\mathrm{D}$ : rearing and feeding compartment

$\mathrm{E}$ : water emptying compartment

a : water inlet

$\mathrm{C}_{1}$ and $\mathrm{C}_{2}$ : removable wall

$d$ : overfloor shoot

$n v$ : water level

$r$ : oxygenation pipe.

Le volume d'élevage est de $\mathrm{I} 6$ litres environ (compartiments B - C - D D) et se trouve limité par deux cloisons amovibles constituées chacune d'une armature de plexiglass de $0,4 \mathrm{~cm}$ d'épaisseur, garnie d'une toile de nylon tressée, à maille carrée de $800 \mu$.

L,es diverses faces des compartiments A, B, D, E, sont en plexiglass de I cm d'épaisseur et sont collées avec de l'altuglass, puis vissées.

La canalisazion $C$ est constituée d'un tuyau de P.V.C. dont les deux extrémités sont soudées au reste du système, grâce à de la colle souple V 26 . 
De plus, afin, d'une part d'éviter la fuite des animaux et d'autre part de maintenir l'équilibre thermique du système, des plaques de verre recouvrent les compartiments $\mathrm{A}, \mathrm{B}, \mathrm{D}$ et $\mathrm{E}$.

Enfin, la structure de cette unité d'élevage, nécessite la mise au point d'un système permettant la capture facile des individus. Après avoir provoqué la fuite des animaux du compartiment $B$, nous introduisons un double disque de plexiglass ajouré dont les pourtours sont garnis de languettes de caoutchouc et la forme épouse parfaitement celle du compartiment $C$. Le disque ainsi introduit est tiré vers le compartiment $D$ entraînant les Civelles (fig. I).

\section{B. - Constance des facteurs physico-chimiques}

\section{Thermorégulation}

Notre élevage en canalisation est réalisé à la température optimum de $25 \pm$ I ${ }^{\circ} \mathrm{C}$ (EIIE, I975; KUhLMANN, I976; Eilie et DAGUzan, I976). Au cours des diverses manipulations (pesées, comptage des individus), les animaux sont toujours maintenus à la température d'élevage.

\section{Oxygénation}

Des contrôles réguliers effectués quatre fois par semaine permettent de constater que le taux de saturation en oxygène est toujours important (90 à IOO p. IOO).

3. $p H$

Il est contrôlé chaque jour et oscille entre 7,5 et 7,7 .

\section{Débit}

Le débit varie entre $5_{5}$ et I $701 / \mathrm{h}$ et est contrôlé matin et soir.

\section{Lumière}

Des panneaux de "polystyrène expansé " sont plaqués sur les fenêtres de la salle d'élevage afin d'uniformiser les intensités lumineuses reçues par la "canalisation n.

\section{C. - Origine des Civelles et dispositions préliminaires}

I es Civelles mises en élevage ont été pêchées le I 5 mars I 976 dans 1'estuaire de la Loire, à l'entrée Esst du port de St-Nazaire (Loire-Atlantique).

Durant le trajet, les animaux sont maintenus dans une enceinte isotherme légèrement humidifiée. 
Les Civelles destinées à l'élevage sont d'abord mises dans un bac dont l'eau présente les caractéristiques de leur milieu d'origine (salinité $20 \mathrm{p}$. Iooo; température $9^{\circ} \mathrm{C}$ ). Puis, les animaux sont amenés progressivement (en $4 \mathrm{j}$ ) aux conditions choisies (eau douce).

Pendant le transfert des animaux des bacs d'adaptation dans les bacs d'élevage, la température est maintenue à $9^{\circ} \mathrm{C}$, pour éviter tout stress éventuel (ELIE et Daguzan, I976). Puis, elle est élevée de 2,5 à $3,5^{\circ} \mathrm{C}$ par jour jusqu'à 1'obtention de la température désirée.

Aucun traitement thérapeutique n'est administré aux Civelles durant 1'expérimentation.

\section{D. - "Charge biotique " des bacs d'élevage}

En généra1, les " charges biotiques " sont calculées par rapport à la surface des bassins d'élevage. Au Japon, elles sont déterminées de façon empirique et varient entre $0,2 \mathrm{~kg} / \mathrm{m}^{2}$ et $0,5 \mathrm{~kg} / \mathrm{m}^{2}$.

En ce qui concerne notre système d'élevage, la charge biotique est exprimée par unité de volume $\left(\mathrm{m}^{3}\right)$. Cette dernière se situe aux environs de $0,865 \mathrm{~kg} / \mathrm{m}^{3}$, charge nettement supérieure à celles communément employées par ailleurs $\left(0,2 \mathrm{~kg} / \mathrm{m}^{3}\right.$ à $\left.0,6 \mathrm{~kg} / \mathrm{m}^{3}\right)$.

\section{E. - Alimentation}

Les besoins alimentaires qualitatifs et quantitatifs ont surtout été étudiés chez Anguilla japonica (TEMrNck et SchlEGEL), (INUI et EGUSA, I966; Nose, I970; ArAi et al., I97I; QUERELLOU, I973; UsUI, I974) mais concernent, soit des Anguillettes (poids supérieur à $2 \mathrm{~g}$ ), soit des Anguilles. En ce qui concerne 1'espèce européenne, Anguilla anguilla, rares sont les travaux relatifs à l'alimentation, et malgré les recherches de MESKE (Ig69) et de SELTZ (I974), le problème reste encore posé pour la phase Civelle.

Pour notre part, nous avons distribué de la rate de bœuf crue et broyée (aliment déjà utilisé dans nos élevages précédents). Celle-ci est disposée dans des mangeoires déjà décrites (EI,IE et DAGUZAN, I976), une fois par jour, généralement entre I 2 heures et IA heures.

\section{F. - Détermination des poids individuels}

Toutes les pesées sont effectuées le matin, à la température de l'eau d'élevage et à l'aide d'une balance "Mettler " ayant une précision de o,or g. Les Civelles sont disposées par groupe de 5 ou ro individus environ sur une feuille de papier filtre et séchées individuellement par un léger mouvement de " roulage ". Nous évitons toute blessure en manipulant à la main.

L'effectif des Civelles au départ de l'élevage est de 72, représentant un poids global ou total de $13,80 \mathrm{~g}$. 


\section{IV. - Présentation et discussion des résultats}

\section{A. - Alimentation des Civelles et comportement alimentaive}

I. Prise de nourriture pendant les 38 premiers jours d'alimentation dans l'élevage en "canalisation"

L'étude de l'évolution du nombre de Civelles ayant un tube digestif dans lequel on distingue la présence d'aliment, pendant les $3^{8}$ premiers jours d'élevage,

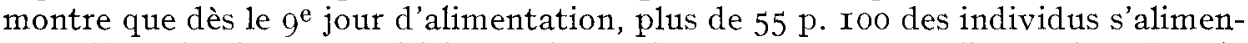
tent (fig. 2). Cette acquisition précoce du comportement alimentaire chez la Civelle confirme nos résultats antérieurs.

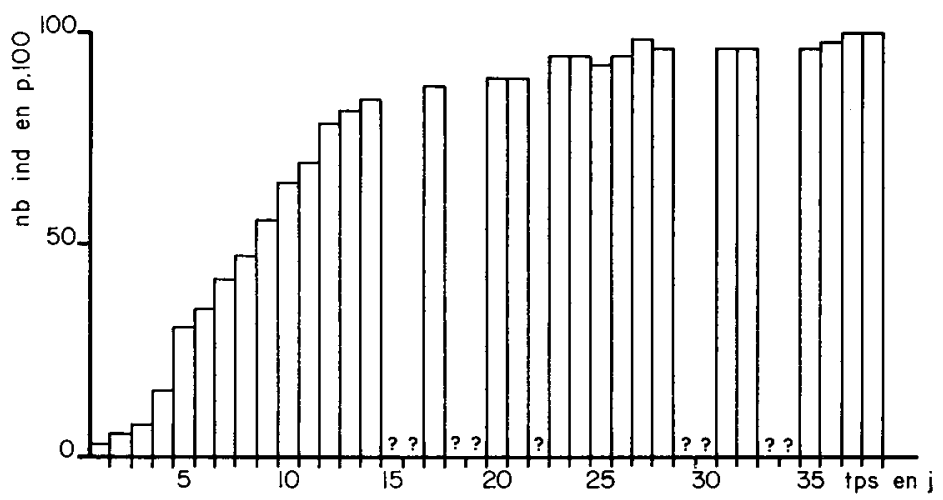

FIG. 2. - Évolution du nombre d'individus s'alimentant dans le système en canalisation pendant les 38 premiers jours d'alimentation; par ropport au nombre d'individus présents.

Variation in the number of individuals consuming food in the canalization system during the first 38 days of feeding as compared to the number of individuals present.

A partir du $I_{7}$ jour, plus de $87 \mathrm{p}$. Ioo des animaux présents s'alimentent.

Le comportement alimentaire présenté par les Civelles puis les Anguillettes correspond à celui que nous avions décrit dans un travail précédent. (EIIE et DAGUZAN, I976). Il faut ajouter que les positions en " $\mathrm{S}$ " sont souvent accompagnées par des mouvements vrillés, lorsque la particule alimentaire est dure et importante.

D'autre part, l'évolution des pourcentages de Civelles se nourrissant par rapport à l'effectif présent au moment des observations montre qu'il existe des individus qui ne s'alimentent jamais. Ces derniers meurent pendant les $3^{8}$ premiers jours (1'amaigrissement étant très important " poids moyen de $0,07 \mathrm{~g}$ ).

\section{Durée moyenne des repas}

Des chronométrages effectués, durant $2 \mathrm{I}$ jours consécutifs (à partir du $38^{\mathrm{e}}$ jour d'alimentation) et pendant la phase d'alimentation montrent que le temps moyen des repas pour l'ensemble des individus est de ro mn environ. Rares sont les 
animaux qui s'alimentent après ce laps de temps. Mais, afin de ne pas créer de facteur limitant, nous maintenons la mangeoire pendant 2 heures, la ration étant ou non utilisée.

\section{La ration alimentaire et son évolution}

Au Japon, il est admis que pour les adultes, il faut distribuer entre 6 p. Ioo (pour un aliment artificiel) et 28 à 30 p. Ioo (pour un aliment naturel) de la biomasse présente dans le système d'élevage (QUERELLOU, I973; UsUI, I974; FORREST, 1976 et 'TESCH, I977). En Europe et pour les adultes, les rations distribuées sont sensiblement les mêmes (C.T.G.R.E.F., I973).

En ce qui concerne nos élevages, le critère retenu pour les augmentations de ration est l'absence totale d'aliment dans la mangeoire au terme des deux heures d'alimentation (fig. 3).

\section{Comportement des Civelles pendant et juste après la phase d'alimentation dans l'élevage en "canalisation"}

L'étude de ces comportements est réalisée grâce à une série d'observations effectuées durant 4 jours $\left(39^{\mathrm{e}}, 4^{\mathrm{e}}, 4 \mathrm{I}^{\mathrm{e}}\right.$ et $4^{\mathrm{e}}$ jour d'alimentation). Le travail consiste à dénombrer au moment de la mise en place de la mangeoire et pendant 1'heure qui suit, toutes les 2, 5 on Io $\mathrm{mn}$, les individus présents dans les différents compartiments đu système d'élevage (fig. 4). Le dénombrement des individus aux moments de grande activité est effectué à l'aide de photographies. Il faut signaler que 40 individus sont présents au moment des différentes observations.

On note ainsi que juste avant l'implantation de la mangeoire, la proportion des individus présents dans le compartiment $C$ est importante ( 27 individus $/ 40$ ); les compartiments $\mathrm{B}$ (oxygénation) et $\mathrm{D}$ (Alimentation) sont faiblement pourvus en individus (au total I3/40).

Dès l'implantation de la mangeoire, il y a une "migration " des Civelles occupant les compartiments $\mathrm{B}$ et $\mathrm{C}$ dans le compartiment $\mathrm{D}$ (30 individus dans les deux premières minutes). Dès la $5^{\mathrm{e}}$ minute d'alimentation, nous assistons à une chute importante des effectifs đu compartiment $D$ au bénéfice, dans un premier temps $\mathrm{d} \mathbf{u}$ compartiment $\mathrm{C}$, puis dans $\mathbf{u n} 2^{\mathrm{e}}$ temps du compartiment $\mathrm{B}$. Enfin, après l'heure d'alimentation, la grande majorité des animaux regagne le compartiment $\mathrm{C}$. Ainsi, cette évolution spatiale s'effectue comme suit

- une phase d'alimentation, relativement brève, ne dépassant généralement pas Io minutes;

- tne phase de transition correspondant au temps mis par les Civelles alimentées pour rejoindre le compartiment $B$;

- une phase d'oxygénation, relativement courte;

- une phase de repos.

Il faut noter que la façon de procéder n'offre qu'une image instantanée du phénomène. Seuls des marquages individuels (très difficiles à ce stade) et des comptages permanents des individus dans les différents compartiments du système permettraient de visualiser complètement les comportements. 


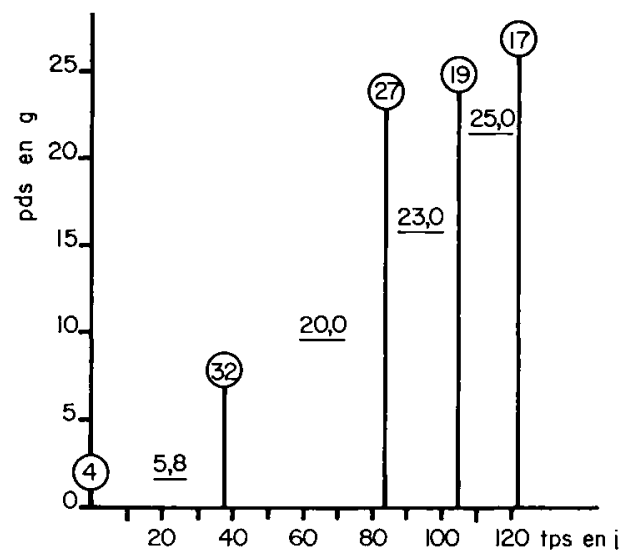

FIG. 3. - Evolution de la ration alimentaire distribuée au cours de l'élevage.

(Les chiffres entourés représentent la ration alimentaire en p. тоo du poids global des individus présents à chaque pesée; ceux soulignés expriment la valeur moyentie de la ration en $\mathrm{g}$ durant chaque période).

\section{Changes in the food ration offered during rearing}

(Encircled figures indicate the food ration in p. roo of the total weight of the individuals present at each weighing; underlined figures indicate the mean value of the ration in $g$ during each period).

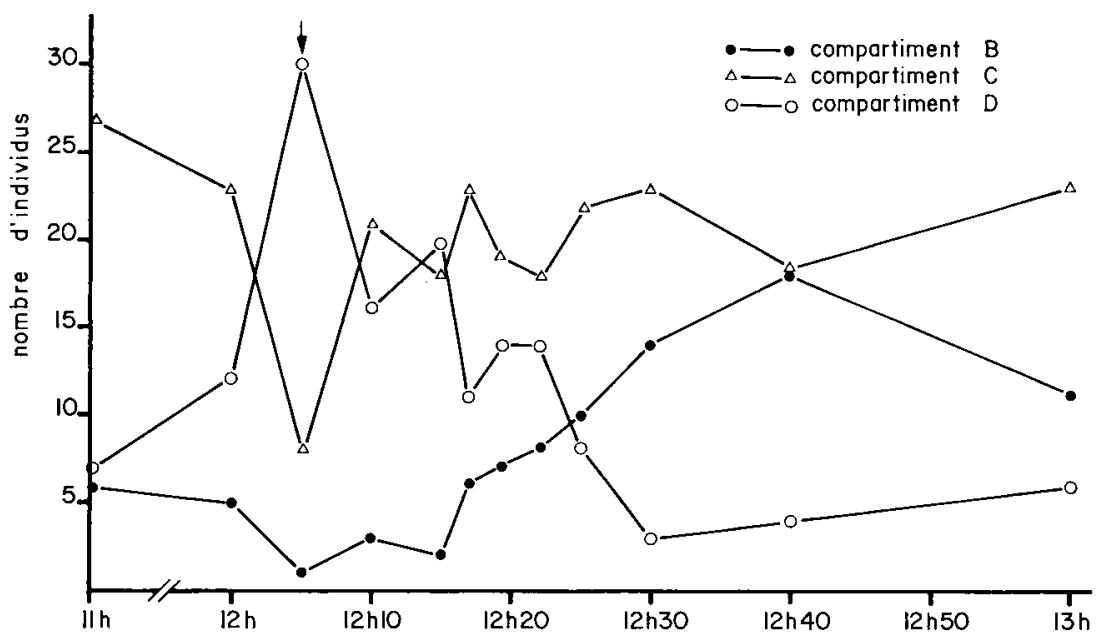

FIG. 4. - Evolution de la distribution spatiale des Civelles dans les compartiments de l'élevage en canalisation pendant la première heure de la phase d'alimentation ( $\downarrow$ moment où l'aliment est distribué).

Variation in the spatial distribution of the glass-eels in the compartments of the canalization system during first hour of the feeding phase ( $\downarrow$ moment of feeding). 
5. Activité et localisation préférentielle des Civelles dans le système d'élevage en canalisation

Cette étude est effectuée sur 3 cycles de 26 heures. Les expériences débutent I heure avant l'implantation de la mangeoire et se terminent le lendemain.

Les observations portent d'une part sur le nombre d'individus actifs et inactifs (sans tenir compte du lieu de leur activité) et d'autre part sur le nombre d'individus présents dans les différents compartiments composant le système (sans tenir compte de leur activité).

\section{a) Activité des Civelles (fig. 5)}

Il existe deux maximum d'activité dus à l'implantation de la mangeoire dans le compartiment $D$.

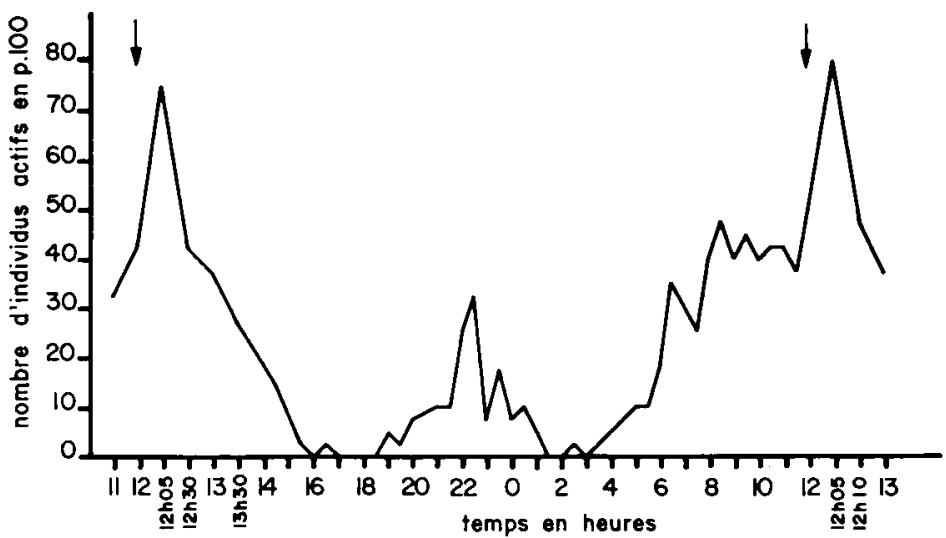

Fig. 5. - Activité des Civelles élevées en canalisation pendant 26 heures consécutives (les flèches indiquent le début de l'alimentation).

Activity of glass-eels reared in canalization during 26 consecutive hours (the arrows indicate the beginning of feeding).

A ces deux pics d'activité, vient s'ajouter un troisième maximum correspondant, semble-t-il, à la tombée de la nuit (2I h) et persistant jusqu'à I heure du matin.

De plus, il faut noter qu'une période de forte activité précède l'implantation de la mangeoire ( $4 \mathrm{~h}$ à $\mathrm{I} 2 \mathrm{~h}$ ), le compartiment $\mathrm{D}$ en est le siège principal.

Enfin, des observations complémentaires permettent de montrer que les individus présents dans la canalisation sont la plupart du temps, inactifs.

\section{b) Localisation préférentielle des Civelles (fig. 6)}

La comparaison entre les distributions spatiales des animaux dans les trois compartiments considérés $(B, C, D)$ permet de penser que : mentation;

- la canalisation ne sert d'abri que pendant la journée après la phase d'ali- 


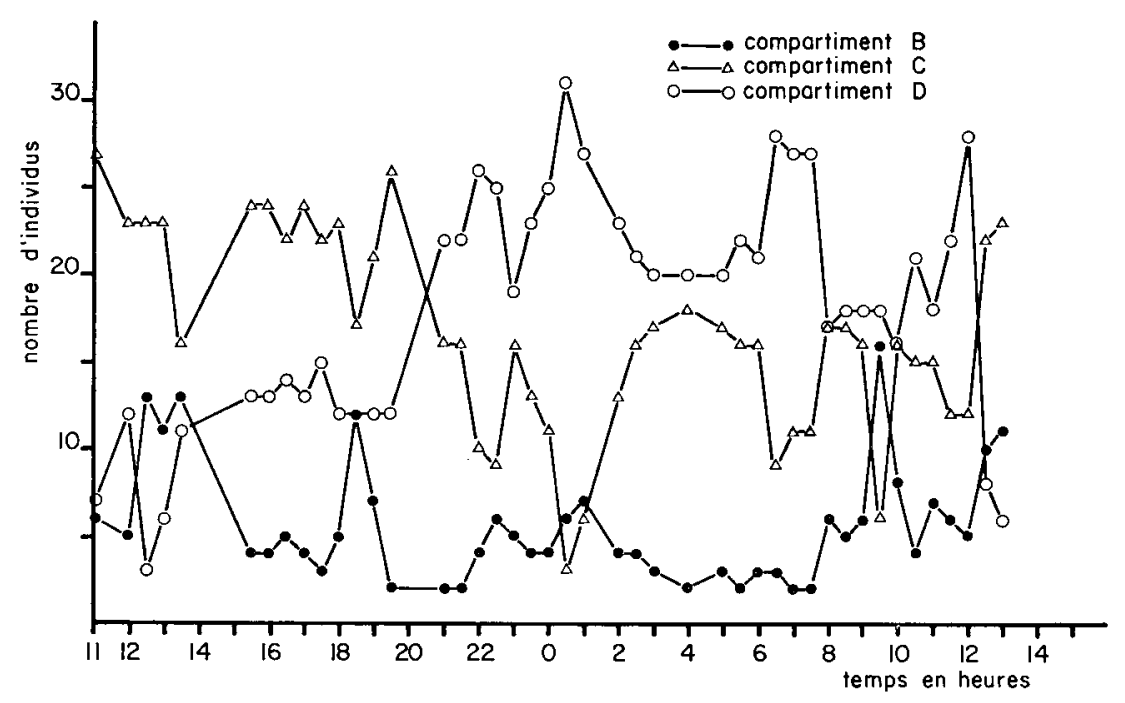

FIG. 6. - Distribution spatiale des Civelles dans les trois compartiments d'élevage du système en canalisation (cycle de $26 \mathrm{~h}$ ). 40 individus sont présents pendant les expérimentations.

Spatial distribution of the glass-eels in the three vearing compartments of the canalization system (26 hr-cycle). 40 individuals were present during the experiments.
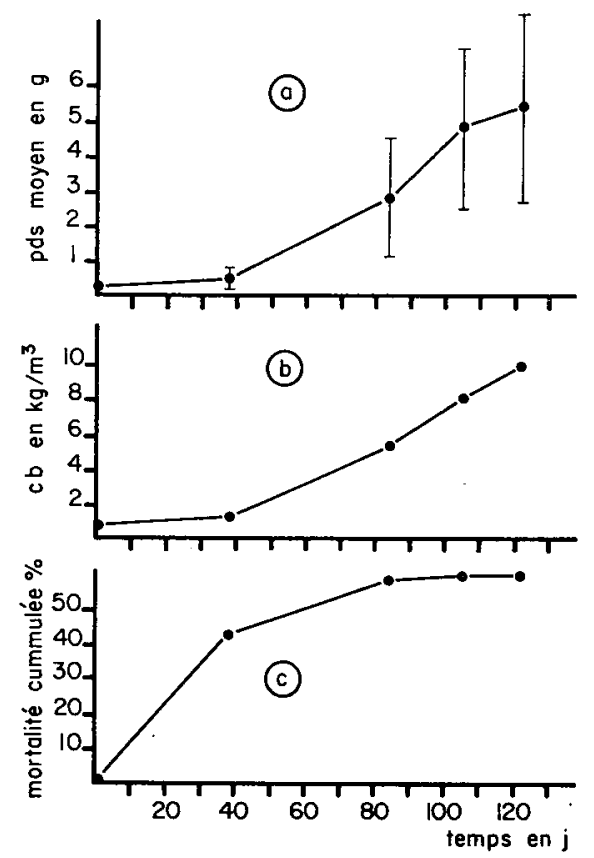

FIG. 7. - Évolution du poids moyen (a), de la charge biotique $(c b),(b)$ et de la mortalité au cours de l'élevage (c)

Changes in mean weight (a), biotic load $(c b),(b)$ and mortality during rearing $(c)$. 
- à partir de 2 I h, il y a un transfert de la plupart des individus du compartiment $\mathrm{C}$ vers le compartiment $\mathrm{D}$ qui est, soit le siège de l'activité nocturne et matinale, soit le siège $\mathrm{du}$ repos pendant les mêmes moments.

- Enfin, aucune conclusion ne peut être tirée en ce qui concerne le compartiment $B$, sauf au niveau du comportement post-alimentaire dont nous avons déjà parlé précédemment.

\section{B. - Croissance des Civelles}

\section{I. Évolution des poids moyen et global au cours de l'élevage en canalisation}

Cette étude correspond à une période de I22 jours d'alimentation (fig. 7).

Tout d'abord, on note que pendant les 38 premiers jours d'alimentation, la croissance est relativement faible, les Civelles atteignent un poids moyen de $0,52 \mathrm{~g}$.

Cependant, dans le même temps, nous avons vu précédemment, que le volume des prises alimentaires est relativement important (fig. 3). Nous pensons donc que ce phénomène est dût essentiellement à deux causes principales :

- 1'ingestion de l'aliment sans croissance (ELIE et DAGUZAN, I976);

- 1'hétérogénéité des lots de départ (E,LIE, I979).

Par la suite, les individus croissent de façon plus importante, le poids moyen passant de $0,52 \mathrm{~g}$ à $5,47 \mathrm{~g}$ (fig. $7^{a}$ ).

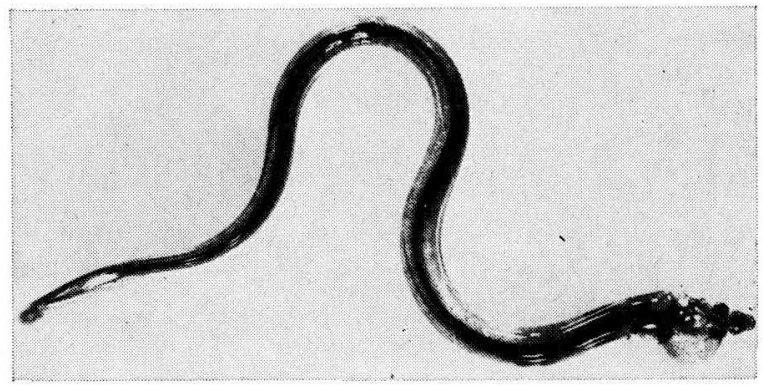

a

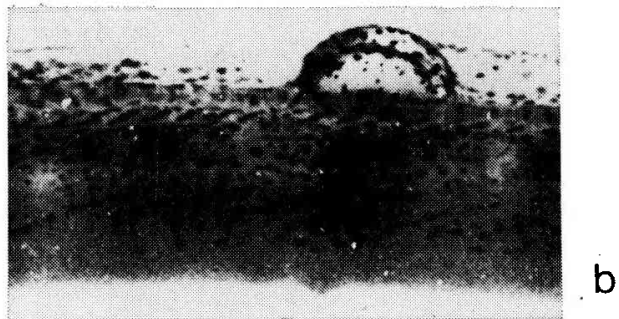

Fig. 8. - Civelles présentant une grosse bulle de gaz sous-épidermique sur le coté droit de la tête (a). Grossissement d'une bulle de gaz $(b)$.

Glass-eels exhibiting a large gas bubble undev the skin on the right side of the head $(a)$. Magnification of a gas bubble $(b)$. 
Le gain de poids global obtenu au terme de l'élevage après I22 jours est de I I5I,0 p. Ioo (passage de I3,80 g à I58,90 g). (fig. $7 b$ ).

La charge biotique passe dans le même temps de $0,86 \mathrm{~kg} / \mathrm{m}^{3}$ à $9,93 \mathrm{~kg} / \mathrm{m}^{3}$

Il faut remarquer que ces valeurs sont nettement supérieures à celles obtenues pour des temps d'élevage équivalents dans les systèmes classiques, aussi bien en eau douce (données non publiées) qu'en eau de mer légèrement dessalée ( $\mathrm{E}_{\mathrm{L}} \mathrm{IE}$ et DaGuZan, I976) pour les mêmes temps d'expérimentation.

Enfin l'évolution des poids globaux suit celle des poids moyens contrairement aux résultats obtenus par SELTZ (I974).

\section{C. - Mortalité et cannibalisme}

I es fuites étant inexistantes, le relevé journalier des individus morts nous permet d'apprécier, d'une part la mortalité périodique et globale, et d'autre part d'établir la valeur du cannibalisme (tabl. r).

Les Civelles présentent des mortalités relativement importantes pendant les premiers jours d'élevage, qui par la suite s'atténuent progressivement (fig. $7 c$ ).

\section{TABLEAU I}

Evolution de la mortalité en $p$. Ioo et du cannibalisme en p. Ioo dans l'élevage en canalisation Development of mortality (in p. soo) and of cannibalism (in p. Ioo) in the canalization rearing system

\begin{tabular}{|c|c|c|}
\hline $\begin{array}{l}\text { Temps } \\
\text { Time }\end{array}$ & $\begin{array}{l}\text { Mortalité } \\
\text { en \% } \\
\text { Mortality } \\
\text { in \% }\end{array}$ & $\begin{array}{c}\text { Cannibalisme } \\
\text { en \% } \\
\text { Cannibalism } \\
\text { in \% }\end{array}$ \\
\hline $\begin{array}{l}\mathrm{I}^{\mathrm{re}} \text { et } 2^{\mathrm{e}} \text { pesée. } \\
\text { Ist and 2nd weighing } \ldots \ldots \ldots \\
\quad 3^{8} \mathrm{j} \quad 3^{8} d\end{array}$ & 37,5 & 5,5 \\
\hline $\begin{array}{c}2^{\mathrm{e}} \text { et } 3^{\mathrm{e}} \text { pesée } \\
\text { 2nd and } 3^{\text {rdd weighing }} \ldots \ldots \ldots \\
4^{6} \mathrm{j} \quad 4^{6} \text { d }\end{array}$ & 12,5 & 2,7 \\
\hline $\begin{array}{l}3^{\mathrm{e}} \text { et } 4^{\mathrm{e}} \text { pesée } . \dot{.} \\
3^{\text {rdd and }} \text { 4th weighing } \\
2 \mathrm{I} \mathrm{j} \quad 2 I d\end{array}$ & $\mathrm{I}, 4$ & o \\
\hline 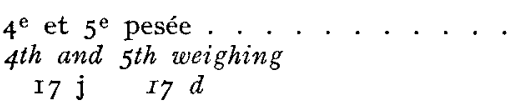 & o & o \\
\hline TOTAL . . . . . . & $5 \mathrm{I}, 4$ & 8,2 \\
\hline
\end{tabular}


Eilles peuvent s'expliquer en partie par le stress des individus transportés en container isotherme, puis soumis à un gradient de température et de salinité. Cependant, nous avons pu montrer que ce stress n'était pas le seul facteur de mortalité. Fin effet, 5 causes principales ont pu être mises en évidence pendant la durée de cette expérimentation (tabl. 2).

Il est intéressant de souligner que l'impossibilité de se nourrir chez quatre individus provient du fait de la formation de bulles de gaz sous l'épiderme dela Civelle (fig. 8). Ces bulles atteignent une importance telle que les individus sont

\section{TABLEAU 2}

Causes des mortalités obtenues chez les individus

élevés dans le système "en canalisation" pendant les I22 jours d'alimentation (exprimées en p. Ioo de l'effectif initial)

Causes of mortality recorded among the individuals reared in the "canalization" system during 122 days of feeding (in p. 100 of initial stock)

\begin{tabular}{|c|c|c|}
\hline \multirow{2}{*}{$\begin{array}{l}\text { Causes des mortalités } \\
\text { Causes of mortality }\end{array}$} & \multicolumn{2}{|c|}{$\begin{array}{l}\text { Bac C } \\
\text { "en canalisation " } \\
\text { "in canalization" }\end{array}$} \\
\hline & $\begin{array}{l}\text { Nombre d'individus } \\
\text { Number of individuals }\end{array}$ & $\%$ \\
\hline Saprolégnioses . . . . . . . & I6 & 22,2 \\
\hline $\begin{array}{l}\text { Individus ne s'alimentant pas } . . \\
\text { Individuals which do not eat }\end{array}$ & 8 & II,$I$ \\
\hline $\left.\begin{array}{l}\text { Cannibalisme. } \\
\text { Cannibalism }\end{array} \cdot \ldots \cdot \ldots \cdot \ldots\right\}$ & 6 & 8,2 \\
\hline $\begin{array}{l}\text { Impossibilité de se nourrir } \\
\text { (bulles de gaz) } \\
\text { Impossibility of food consumption } \\
\text { (gas bubbles) }\end{array}$ & 4 & 5,5 \\
\hline $\begin{array}{l}\text { Impossibilité de défécation } . \\
\text { Impossibility of defecation }\end{array}$ & 2 & 2,7 \\
\hline $\begin{array}{l}\text { Causes indéterminées } \\
\text { Miscellaneous }\end{array}$ & 7 & 9,7 \\
\hline TOTAL. . . . . . & 43 & 59,4 \\
\hline
\end{tabular}


astreints à nager en surface, sans parvenir à s'enfoncer, pour accéder à la mangeoire (la position de l'animal dépend des lieux de formation des bulles de gaz).

De plus, les Civelles présentant une impossibilité de défécation ont généralement une paroi fibreuse (longueur de $0,5 \mathrm{~cm}$ à $6 \mathrm{~cm}$ ) de rate de bœuf qui obstrue 1'orifice anal. Elles s'alimentent quelques jours et meurent ensuite avec un estomac très ballonné.

Quoiqu'il en soit, les mortalités obtenues en " canalisation " sont importantes (43 individus $/ 72$ ). La majeure partie d'entre elles (37,7 p. I00) est due aux Saprolégnioses (I6/43). Sans celles-ci, le taux de survie de cet élevage atteindrait pendant la première période ( 38 jours) 80 à $85 \mathrm{p}$. Ioo.

Les valeurs du cannibalisme présentées chez les Anguillettes au cours de cette expérimentation, semblent être plus importantes que celles obtenues dans un élevage de type classique (EIIE et DAGUZAN, I976). En fait, il est rare de pouvoir observer un phénomène de cannibalisme dans un élevage expérimental (3 cas observés au cours de l'ensemble de nos expérimentations). La présence d'une semiobscurité permanente dans le compartiment $\mathrm{C}$ semble favoriser ce phénomène.

\section{Conclusion}

I a mise au point d'un nouveau système d'élevage, dit " en canalisation " permet d'obtenir chez les Civelles des croissances importantes. En effet, la charge biotique initiale étant de $0,865 \mathrm{~kg} / \mathrm{m}^{3}$ (poids moyen individuel de $0,20 \mathrm{~g}$ ) atteint après I05 jours d'alimentation près de $9 \mathrm{~kg} / \mathrm{m}^{3}$ (poids moyen individuel de $4,87 \mathrm{~g}$ ). Après I22 jours, le système supporte une charge de Io $\mathrm{kg} / \mathrm{m}^{3}$ (poids moyen individuel de $5,47 \mathrm{~g}$ ). Ces résultats sont beaucoup plus importants que ceux obtenus expérimentalement dans des systèmes classiques, pour des durées identiques d'élevage.

L'intérêt de ce système réside dans la disposition relative des différents compartiments (compartiments d'oxygénation ou " respiroir ", "dortoir ", compartiment d'alimentation), les deux premiers cités n'étant jamais souillés par les particules alimentaires.

La compartimentation de cette unité d'élevage a permis de montrer d'une part, 4 phases comportementales successives : alimentation, "transition ", oxygénation, repos, et d'autre part, l'existence selon le moment de la journée, d'une localisation préférentielle des Civelles dans les différents compartiments. Le tube obscur sert surtout d'abri et de repos pendant le jour et après la période d'alimentation.

On aurait pu penser que l'action conjuguée de l'éloignement " respiroir " compartiment d'alimentation, et $\mathrm{d} u$ sens $\mathrm{du}$ courant existant dans le système auraient entraîné des perturbations dans la perception de l'aliment distribué aux Civelles. En fait, il n'en est rien, car dès le $9^{\mathrm{e}}$ jour, plus de $55 \mathrm{p}$. roo des individus s'alimentent et au $\mathrm{I} 7^{\mathrm{e}}$ jour, $87 \mathrm{p}$. roo des Civelles prennent un repas.

Durant cet élevage, 5 causes de mortalité ont pu être déterminées : les Saprolegnioses (22 p. I0o), le cannibalisme ( 8 p. Ioo), les individus ne s'alimentant pas (II p. IOo), ne pouvant se nourrir (bulle de gaz) $(5,5$ p. IOo), ou incapables de rejeter leur fèces (3 p. Ioo). Enfin il faut noter que la présence d'une semi-obscurité permanente dans l'un des compartiments de l'unité d'élevage semble favoriser le cannibalisme. 


\section{Remerciements}

Nous tenons à remercier Madame A. Mr. ÉLIE, assistante au laboratoire des services vétérinaires de Rennes, pour la détermination des Saprolegnioses.

\section{Summary}

\section{Feeding and growth of glass-eels Anguilla Anguilla I. (Anguiliforme Teleosts) reared experimentally in a "canalization" at the laboratory}

Development of a new rearing system using a canalization has led to high growth performances in glass-eels. The initial biotic load (stocking rate) was $0.865 \mathrm{~kg} / \mathrm{m}^{3}$ (individual mean weight: $0.20 \mathrm{~g}$ ) and reached after 105 days of feeding almost $9 \mathrm{~kg} / \mathrm{m}^{3}$ (individual mean weight: $4.87 \mathrm{~g}$ ). After $\mathrm{I} 22$ days, the system supported a load of $\mathrm{ro} \mathrm{kg} / \mathrm{m}^{3}$ (individual mean weight: $5.47 \mathrm{~g})$. These results are much better than those recorded in classical systems for the same length of culture.

The advantage of this system depends on the relative location of the different compartments (oxygenation compartment or "breathing room", "dormitory", feeding compartment), the first two being never polluted by food particles.

The division of this system into compartments permitted to demonstrate 4 successive behavioural phases: feeding, "transition", oxygenation, rest and the existence of a preferential localization of the glass-eels in the different compartments according to the moment of the day. The dark tube was used especially as a shelter and a rest room during the day and after feeding.

It was assumed that the combined action of the distance between the " breathing-room" and the feeding compartment and the direction of the water flow in the system would lead to a disturbance of the perception of the food offered to the glass-eels. But, this was not the case as more than $55 \mathrm{p}$. Ioo were eating already from the gth day and on day 17,87 p. roo of the fishes were consuming a meal.

Five causes of mortality were recorded during this experiment, i.e. saprolegnioses ( 22 p. Ioo), cannibalism ( 8 p. Ioo), individuals which were not eating (I I p. Ioo) unable to eat (gas bubble) or unable to reject their faeces $(3 \mathrm{p}$. Ioo). It has also to be noticed that the permanent semiobscurity in one of the compartments seemed to favour the canibalism.

\section{Références bibliographiques}

Arai S., Nose T., Hashmoto Y., I97I. A purified test diet for the eel. Anguilla japonica. Bull. Freshwater fish. Res. Lab., 21, 2, т6т-т 78 .

BoETIUS J., 1962. Studies of ovarial growth induced by hormone injections in the European and American eel (Anguilla anguilla $\mathrm{L}_{\text {.) }}$ ) and (Anguilla rostrata Le Sueur). Meddr. Danm. Fisk. -og Havunders., 3 (7-12), 183-198.

C.T.G.R.E.F., I973. Développement de l'élevage de l'Anguille en France. Rapport Bordeaux, 24 p. CUEFF J. C., I974. L'élevage de l'Anguille française au Japon. Pêche maritime, 1153, 238-243.

ĖIIE P., I975. Élevages expérimentaux de la Civelle d'Anguilla anguilla $\mathrm{L}$. I $75^{8}$, en eau saumâtre; résultats préliminaires. D.E.A., Univ. Rennes, 73 p.

Eitre P., T979. Contribution à l'étude des montées de Civelles d'Anguilla an guilla Linné (Poisson Téléostéen Anguilliforme) dans l'estuaire de la Loire : Pêche, Écologie, Écophysiologie et Élevage. Thèse doc. $3^{\mathrm{e}}$ cycle, Rennes, $38 \mathrm{I} \mathrm{p}$.

EliE P., Daguzan J., I976. Alimentation et croissance des Civelles d'Anguilla anguilla L. (Poisson Téléostéen Anguilliforme) élevées expérimentalement à diverses températures, au laboratoire. Ann. Nutr. Alim., 30, 95-II4.

PORREST D. M., I976. Eel capture, culture, processing and marketing., ed. Fishing News Book, London, $205 \mathrm{p}$.

Fon'taine M., 1936. Sur 1a maturation complète des organes génitaux de 1'Anguille mâle et l'émission spontanée. C. R. hebd. Séanc. Acad. Sci. Paris, 202, 1312-13r4. 
Fontaine, M., Bertrand E., Lopez E., Callamand O., i964. Sur la maturation des organes génitaux de l'Anguille femelle ( Anguilla anguilla) et l'émission spontanée des cufs en aquarium. C. R. hebd. Séanc. Acad. Sci. Paris, 259, 2907-2910.

InUI Y., EguSA S., I966. Enlarged liver of eel kept on a formulated eel feed. Annual meeting Jap. Soc. Sci. Fish. in ARAI et al., r971.

ITOSHI H., 1972. On the culture of imported European Glass-eel. Bull. Shizuoka Pref. Fish. Exp. Stat., 5, 57-64.

Koops H., I966. Ein Futterungsversuch mit Salzaalen in der Flussteich-wirtschaft Müden an der Mosel. Arch. Fishwiss., 17, 36-44.

Koops H., 197r. Ergebnisse einer Futterung von Teichaalen mit Frischfish und mit Trockenfutter. Fischwirt., a1, 73-77.

KUhlmann H., I975. Der EinfluB von temperatur, Futter, röBe und Herkunft auf die Sexuelle Differenzierung von Glasaalen (Anguilla anguilla). Helgoländer wiss. Meeresunters., 27, I39I 55 .

KUHLMANN H., 1976. Influence of temperature, food, size and origin on the growth and sexual differentiation of elvers (Anguilla anguilla). I.C.E.S./E.I.F.A.C. Symp. on Eel Res. and Management. I2, Helsinki.

Matsur I., I952. A guide to pond-culture of the eel. (En japonais). Journ. Shimonoseki Fish. coll., 2, I-245.

Meske C. H., I969. Aufzucht von Aalbrut in Aquarien. Arch. Fischwiss., 20, 26-32.

Nose T., I970. A preliminary report on some essential amino acids for the growth of eel, $A n g u i l l a$ japonica. Bull. Freshwater Fish. Res. Lab., 19, 31-36.

QUERELlou J., I973. Élevage des Anguilles au Japon. Rapport, C.T.G.R.F.F., Bordeaux.

QUERELLOU J., I975. Facteurs limitants de l'élevage des Anguilles en France. Techniques japonaises correspondantes et " adaptabilité ». Bull. Soc. Franco-Japonaise. Océanogr., (La mer), 13, 30-37.

SEITZ J., I974. Élevage de Civelles en civcuit fermé. Test de grossissement. Rapport C.T.G.R.E.F., Bordeaux.

TESCH F. W., 1977. The eel, biology and management of anguillid eels. ed. Chapman and Hall, Iondon, $434 \mathrm{p}$.

Tuzet O., Fontaine M., I937. Sur la spermatogénèse de l'Anguille argentée (Anguilla vulgaris Cuv.). Arch. Zool. exp. Gen. Paris., 78, I99-1 25.

Usur A., 1974. Eel culture. ed. Fishing news book, London, i 85 p. 